\title{
Italy outsources peer review to NIH
}

The US National Institutes of Health (NIH) is gearing up to begin a review of about 1,000 biomedical research grant applications for the Italian government, an experimental collaboration that comes at an inconvenient time for the US funding agency.

The outsourcing agreement was made last year at the request of Ferruccio Fazio, now Italy's deputy minister for health in the welfare ministry, who is looking to improve the department's peer-review system for awarding competitive research grants (see Nature 455,$719 ; 2008$ ). But its impact comes as the NIH deals with a flood of applications of its own, triggered by the US economic stimulus package (see Nature 459, 763; 2009).

"We took on this project before the Recovery Act was passed, and we never would have taken on the Italian applications if we had known what our workload would be now," says Antonio Scarpa, director of the NIH's Center for Scientific Review. "Nonetheless, we are honoured to assist the Italians." It is the first time the NIH has provided systematic technical support for another country's grant applications, he says.

Most biomedical research funds in Italy are dispensed through government appropriations to institutions, not through a competitive grant system. Many of the national peer-review systems that do exist, and which work in response to irregular funding calls, are plagued by accusations of conflicts of interest among a small pool of reviewers.

"We want to change the culture. We need a peer-review process that is more transparent, and less prone to suspicions of bias," says Giovanni Lucignani, a diagnostic-imaging specialist at the University of Milan.

Jacopo Meldolesi, a neuroscientist at VitaSalute San Raffaele University in Milan who ran a competitive grant programme in Italy using foreign reviewers, argues that the process might work better with Italian scientists participating in the NIH review committees.
"My reservation is that this is being done outside the Italian community," he says.

The funding at stake will provide about $€ 29$ million (US $\$ 40$ million) to support 50 to 60 projects from young researchers; funds will be distributed in January 2010.

Scarpa says the NIH has agreed with the Italian government to delay the start of reviews until it has finished Recovery Act applications. "After we help coordinate reviews this year, we expect the Italians will be ready to run things on their own," he says.

Robert Paul Königs, head of scientific affairs at Germany's main funding agency, the DFG, adds that asking foreign scientists to review individual proposals is standard practice for many funding agencies. "In 2007, some $22 \%$ of reviewers consulted by the DFG were based outside Germany," he says. "But I am not aware of the evaluation of a complete set of proposals turned over to an outside agency."

Richard Van Noorden 